\title{
Archéopages
}

Archéopages

Archéologie et société

Hors-série 1 | 2008

Constructions de l'archéologie

\section{Néolithique en Ile-de-France : la révolution reste à faire}

Anaïck Samzun, Richard Cottiaux et Yves Lanchon

\section{(2) OpenEdition}

\section{Journals}

Édition électronique

URL : https://journals.openedition.org/archeopages/885

DOI : 10.4000/archeopages.885

ISSN : 2269-9872

Éditeur

INRAP - Institut national de recherches archéologiques préventives

Édition imprimée

Date de publication : 1 février 2008

Pagination : 124-126

ISSN : 1622-8545

\section{Référence électronique}

Anaïck Samzun, Richard Cottiaux et Yves Lanchon, « Néolithique en lle-de-France : la révolution reste à faire », Archéopages [En ligne], Hors-série 1 | 2008, mis en ligne le 01 février 2008, consulté le 26 février 2023. URL : http://journals.openedition.org/archeopages/885 ; DOI : https://doi.org/10.4000/ archeopages.885 


\begin{tabular}{|c|c|c|c|c|c|c|}
\hline Étapes chronoculturelles & $\begin{array}{l}\text { Dates } \\
\text { (av.n.e.) }\end{array}$ & $\begin{array}{l}\text { Sépultures } \\
\text { individuelles }\end{array}$ & $\begin{array}{l}\text { Nécropoles } \\
\text { et sépultures } \\
\text { collectives }\end{array}$ & Habitats & Bâtiments & $\begin{array}{l}\text { Minières et } \\
\text { ateliers }\end{array}$ \\
\hline $\begin{array}{l}\text { Néolithique ancien } \\
\text { Rubané final du Bassin parisien }\end{array}$ & -5000 & 6 & o & 4 & 2 & o \\
\hline $\begin{array}{l}\text { Néolithique ancien } \\
\text { Villeneuve-Saint-Germain }\end{array}$ & -4900 & 26 & o & 55 mini & 21 & o \\
\hline $\begin{array}{l}\text { Néolithique moyen I } \\
\text { Cerny }\end{array}$ & -4600 & \pm 80 & 3 & $50 ?$ & o & $?$ \\
\hline $\begin{array}{l}\text { Néolithique moyen II } \\
\text { Roessen tardif, post-Roessen } \\
\text { et Chasséen ancien }\end{array}$ & -4400 & $\mathrm{o} ?$ & o? & 3 & $1 ?$ & $?$ \\
\hline $\begin{array}{l}\text { Néolithique moyen II } \\
\text { Michelsberg/Chasséen/ } \\
\text { groupe de Noyen }\end{array}$ & -4200 & \pm 35 & 2 & 18 & o & 3 \\
\hline $\begin{array}{l}\text { Néolithique moyen II et III } \\
\text { Groupe de Balloy/Gravon }\end{array}$ & $-3700 ?$ & 11 & 1 & $3 ?$ & o & 1 \\
\hline $\begin{array}{l}\text { Néolithique récent } \\
\text { Ex-Seine-Oise-Marne }\end{array}$ & -3400 & $?$ & 81 & 28 & 1 & $3 ?$ \\
\hline $\begin{array}{l}\text { Néolithique final } \\
\text { Gord }\end{array}$ & -2900 & o & $10 ?$ & 21 & o & $1 ?$ \\
\hline $\begin{array}{l}\text { Néolithique final } \\
\text { Campaniforme, } \\
\text { Épicampaniforme }\end{array}$ & $\begin{array}{l}-2500 \\
\text { à } \\
-2100\end{array}$ & 3 & $6 ?$ & 11 & o & $?$ \\
\hline
\end{tabular}

[Tab.1] État de la documentation

sur le Néolithique en Île-de-France (tous types de fouilles confondus). 


\section{Néolithique en Ile-de-France : la révolution reste à faire}

\author{
Anaïck Samzun \\ Richard Cottiaux \\ Yves Lanchon \\ Inrap, UMR 7041 «Archéologies et Sciences de l'Antiquité» \\ Je tiens beaucoup de ma mère, \\ Et papa m'implique \\ Je suis dans l'âge de pierre, \\ Paléolithique. \\ Mais on me dit à la ronde \\ Pas méchant, doué \\ Et dans l'âge du Bronze \\ J’ai déjà un pied...V.S.Vyssotski
}

L'ouvrage La Révolution néolithique en France sous la direction de J.-P. Demoule est paru en 2007. Il fait le point sur les dernières recherches et en dresse un tableau synthétique. On y apprend notamment que les connaissances relatives au Néolithique ont fortement progressé depuis le développement de l'archéologie préventive. Les éléments-clés du cadre chronoculturel sont désormais fixés. La périodisation a même parfois atteint un degré de précision suffisant pour suivre l'évolution des premières sociétés paysannes. Mais ces avancées ont également l'avantage de mettre en exergue des lacunes, des imprécisions et des questions irrésolues sur cette période. Ce sont ces lacunes qui nous intéressent ici. Il existe une image plus prosaïque des avancées de la recherche sur le Néolithique que nous avons choisi de dessiner à une échelle régionale, en prenant l'Ile-de-France comme exemple.

Au premier abord, l'impression est positive. On constate depuis une trentaine d'années une très forte activité archéologique liée au nombre d'aménagements du territoire. Elle est renforcée par l'existence d'un tissu de chercheurs œuvrant dans le cadre de programmes de recherches (Bassée, vallée de la Marne...). Aussi nombre de sites néolithiques ont-ils été découverts au cours d'opérations menées sur de larges superficies, révélant de multiples structures riches en mobilier, des plans de bâtiments et d'enceintes, ainsi que de nombreuses sépultures. Le cadre chronoculturel du Néolithique se décline à l'échelle de la région en neuf étapes de -5100 à -2100 , soit une durée de 3000 ans [Tabl.1].

Le Néolithique francilien n'est pas moins connu que dans d'autres régions, la place qu'il occupe est bien déterminée et ses spécificités sont identifiées.

En seconde analyse, la mise en perspective des données de terrain pour chaque étape du Néolithique permet de dévoiler une autre réalité.

D'abord, un faible niveau de redondance. Le nombre de sites majeurs (comme Balloy «les Réaudins», Jablines «la Pente de Croupeton», Noyen-sur-Seine «les Hauts des Nachères»...) est restreint et concerne surtout les périodes anciennes et moyennes du Néolithique.

Le reste de la documentation comprend soit des occupations partiellement fouillées ou représentées par quelques fosses, soit des indices. Seules trois enceintes ont été intégralement décapées et aucun plan entier de village ou de nécropole n'est connu. Les bâtiments sont rares et ne concernent tout au plus que quatre étapes du Néolithique sur neuf, principalement les plus anciennes. Cela implique que l'analyse de l'importance et de l'organisation des groupes humains est encore difficile à mener.

Ensuite, on est en mesure d'identifier les hiatus documentaires, dont le plus flagrant s'intercale entre le Néolithique moyen et le Néolithique récent.

Enfin, il subsiste des déséquilibres géographiques entre vallées et plateaux, et entre départements (la moitié est de la région ayant livré plus des deux tiers des données, dont tous les sites majeurs). Puisque la densité du peuplement est forte dès la fin du Néolithique ancien, c'est donc un défaut de recherches dans les autres secteurs qui est mis en lumière aujourd'hui. Le Néolithique est une trame dont les vides l'emportent sur les pleins.

Finalement, la révolution sur le Néolithique reste à faire. D'un côté, les grands projets d'aménagement: villes nouvelles, Zac, etc., sont en constant développement. Leur programmation pèse désormais sur toute l'Ile-de-France.

De l'autre, avec une dizaine de néolithiciens, si la région semble mieux lotie que d'autres, le nombre d'acteurs reste très insuffisant face aux nouvelles exigences. La progression des aménagements entraîne un changement d'échelle: c'est la région qui devient l'assise des nouveaux programmes de recherche. Après la phase de croissance de l'archéologie préventive, on conçoit comme un atout cette mise en évidence des lacunes car elle permet désormais d'affiner significativement les problématiques existantes. C'est une nouvelle situation qui implique donc de véritables défis:

- méthodologiques, avec la caractérisation des sites et des structures, même les plus fugaces; diagnostics en tranchées étroites de 2 mètres et limitations des zones à fouiller devront être sérieusement rediscutés;

- organisationnels, avec la constitution d'une équipe régionale, la présence de néolithiciens sur les diagnostics, la formation de néolithiciens et la diffusion de l'information auprès des autres archéologues:

- administratifs et politiques: il faut convaincre aménageurs et, parfois, tutelles de l'intérêt des sites peu structurés qui représentent en fait l'essentiel de l'information; à défaut, le risque est d'occulter les deux tiers des étapes du Néolithique;

- scientifiques: outre deux programmes de recherche intégrant des données franciliennes (ACR Néolithique ancien en vallée de Marne, PCR III millénaire), il est impératif de combler le retard de publication des données de terrain. 
L'exemple francilien n'est sans doute pas unique. Il fait déjà écho à une situation analogue en Picardie (Dubouloz et al. 2005). En bref, si la révolution néolithique a eu lieu en 5000 avant notre ère, celle des études sur le Néolithique se doit d'être permanente...

Constantin C., Mordant D., SimoninD. 1997: La Culture de Cerny, nouvelle économie, nouvelle société au Néolithique, actes du colloque international de Nemours, 9-11 mai 1994, Nemours, ApRAIF.

Chambon P., Mordant D., Pariat J.-G. 2003: «Sépultures du Néolithique moyen en Bassin parisien: le cas des architectures sépulcrales», in Actes du colloque interrégional sur le Néolithique, Luxembourg, p.9-31.

Demoule J.-P. (dir.) 2007: La Révolution néolithique en France, La Découverte, Paris.

Dubouloz J., Bostyn F., Chartier M., Cottiaux R., Le Bolloch M. 2005: «La recherche archéologique sur le Néolithique en Picardie», in «La recherche archéologique en Picardie: bilans et perspectives, journées d'études tenues à Amiens les 21 et 22 mars 2005", Revue archéologique de Picardie, $\mathrm{n}^{\circ} 3-4$, p.63-98.

\section{Sur la genèse de la convention de Malte}

Willem J. H. Willems

Université de Leyde, faculté d'Archéologie

$\mathbf{L}$ es différents systèmes de mise en application de la Convention européenne sur la protection du patrimoine archéologique ${ }^{\mathbf{1}}$ font, depuis plusieurs années, l'objet de débats au sein de la communauté archéologique. Indépendamment de la manière dont elle est appliquée par les différents pays, il est clair que la convention de Malte a occasionné partout des changements considérables dans la pratique de l'archéologie.

Des différences subsistent entre les systèmes entièrement ou partiellement privatisés et ceux dans lesquels l'État exerce encore un monopole sur l'archéologie. Mais les changements fondamentaux ont été les mêmes partout en Europe. Les archéologues universitaires sont à présent minoritaires; quasiment toute l'archéologie - y compris dans les systèmes non commerciaux - se pratique aujourd'hui sous contrat, d'où une inquiétude générale relative à sa pertinence, voire à sa qualité intrinsèque (Willems, Van den Dries 2007). Dans le même temps, les ressources de l'archéologie en général ont atteint des niveaux sans précédent, la conservation in situ des sites archéologiques étant devenue dans bien des cas une option réaliste qui répond à un intérêt croissant du public.

Le mérite en revient directement ou indirectement au succès politique de la convention de Malte et aux effets de son application.

La création de cette convention ne doit évidemment rien au hasard. Dans ce texte, il me semble intéressant d'évoquer brièvement les circonstances qui ont précédé sa signature en 1992, les raisons qui l'ont motivée et la façon dont les choses se sont déroulées. ${ }^{2}$

L'historique de la convention. En 1988, le Conseil de l'Europe fondait un nouveau comité intitulé «Comité restreint d'experts du Conseil de l'Europe sur l'archéologie et l'aménagement». Il avait pour mission de préparer une révision de la convention de Londres de 1969, qui avait été la première convention européenne pour la protection du patrimoine archéologique, mais qui était perçue comme inefficace et inadaptée face aux nouveaux défis rencontrés par l'archéologie dans les années 1980.

L'origine de ce développement remonte aux années 1960, lorsque les préoccupations environnementales acquirent de l'importance à la suite de la publication du rapport du Club de Rome. Très vite, il était apparu que le danger qui menaçait les ressources naturelles pesait également sur les ressources culturelles, lesquelles nécessitaient une gestion particulièrement attentive. Cette prise de conscience donna naissance à la gestion des ressources archéologiques dans le sens moderne du terme. Dans l'ensemble du monde occidental, les notions alors dominantes de préservation historique par le biais de la protection des monuments anciens et des «antiquités nationales» ont graduellement cédé le pas à des conceptions plus dynamiques d'une gestion des ressources archéologiques intégrée dès le départ aux systèmes de planification de l'espace urbain et rural sujet à des transformations de plus en plus rapides. Parti des États-Unis dans les années 1970, le mouvement a gagné une décennie plus tard de nombreux pays européens; depuis, il s'est répandu un peu partout dans le monde.

En Europe, la menace que l'effort de reconstruction de l'après-guerre faisait peser sur les ressources archéologiques eut initialement pour réponse des fouilles de sauvetage, entreprises sur une petite échelle et concernant surtout les édifices religieux et les centres urbains détruits par les bombardements. Ces fouilles-là ne différaient guère des fouilles programmées menées à l'époque par des institutions de recherche. Avec l'essor économique des années 1960 et 1970, cette stratégie aboutit à des fouilles de sauvetage sur une échelle sans précédent, accompagnant les projets de développement des infrastructures partout en Europe. Ce phénomène se poursuivit dans les années 1980, avec de puissantes organisations, agissant généralement sous le contrôle de l'État, engagées dans d'ambitieux chantiers archéologiques qui dominaient le travail sur le terrain. Ce travail, qui nécessitait des moyens importants, était la plupart du temps financé par les gouvernements, bien que, à l'occasion, quelques fonds puissent être alloués par les aménageurs.

Cependant, dans les années 1980, on commença à prendre conscience de la disparition rapide des ressources archéologiques sous les bulldozers: les fouilles de sauvetage ne pouvaient collecter qu'une infime partie de l'information, le reste étant irrémédiablement perdu. La profession reconnut alors la gravité de la menace pesant sur le patrimoine archéologique et prit conscience du besoin 